\title{
Reflets
}

Revue d'intervention sociale et communautaire

\section{Minorité sexuelle et individu : l'intervention auprès des femmes homosexuelles dans les soins de santé}

\section{Sabina Grabowiecka}

Volume 17, numéro 2, automne 2011

URI : https://id.erudit.org/iderudit/1012134ar

DOI : https://doi.org/10.7202/1012134ar

Aller au sommaire du numéro

Éditeur(s)

Reflets, Revue d'intervention sociale et communautaire

ISSN

1203-4576 (imprimé)

1712-8498 (numérique)

Découvrir la revue

Citer cet article

Grabowiecka, S. (2011). Minorité sexuelle et individu : l'intervention auprès des femmes homosexuelles dans les soins de santé. Reflets, 17(2), 163-173.

https://doi.org/10.7202/1012134ar

Tous droits réservés (C) Reflets, Revue d'intervention sociale et communautaire, 2012
Cecument est protégé par la loi sur le droit d'auteur. L'utilisation des services d'Érudit (y compris la reproduction) est assujettie à sa politique d'utilisation que vous pouvez consulter en ligne.

https://apropos.erudit.org/fr/usagers/politique-dutilisation/ 


\title{
Minorité sexuelle et individu : l'intervention auprès des femmes homosexuelles dans les soins de santé
}

\author{
Sabina Grabowiecka
}

Maîtrise en service social

\section{Introduction}

La discrimination envers les personnes de minorité sexuelle est toujours présente et requiert une attention particulière, surtout en ce qui concerne la qualité des services de santé qui leur sont offerts.

En tant que profession, le service social dirige son action vers un changement à la fois individuel et social, et ce, dans une optique de justice sociale.Ainsi, une large part du travail des intervenantes et intervenants consiste à revendiquer les droits de minorités qui sont parfois victimes de discrimination sous diverses formes. Cette orientation professionnelle qui consiste à venir en aide aux personnes issues de groupes minoritaires et à défendre leurs droits renferme cependant un double piège pour les intervenantes ou intervenants : d'abord, le manque de compréhension en présumant des besoins de ces personnes et en leur imposant des valeurs ou croyances personnelles ou professionnelles, puis le fait de référer à un groupe minoritaire — « les immigrants », "les homosexuels » "les jeunes ", etc. — en imposant une vision homogène de celles 
ou de ceux qui en font partie et en laissant de la sorte peu de place à la reconnaissance de leur individualité. Alors, comme ils appartiennent surtout au groupe majoritaire, comment les intervenantes et intervenants peuvent-ils en arriver à défendre efficacement les droits des groupes minoritaires auprès desquels ils s'engagent? Convaincus qu'ils sont de la légitimité de leurs actions et de la grandeur des valeurs sur lesquels elles se fondent, comment peuvent-ils éviter le piège du "professionalo-centrisme "?

Dans le but de répondre à ces deux questions et d'ainsi contribuer à l'amélioration des services offerts aux personnes appartenant à une minorité sexuelle, j’ai rédigé un mémoire de maîtrise ${ }^{1}$ portant sur l'accès des femmes homosexuelles à des soins de santé. Quelques éléments saillants de ma recherche, de ma réflexion et de mes pistes de recommandations font l'objet du présent article.

\section{Le coming out, pourquoi faire?}

La discrimination basée sur une identité autre qu'hétérosexuelle - homosexuelle, queer, bisexuelle, transsexuelle, lesbienne, etc. - est une forme d'injustice sociale toujours présente dans nos sociétés. Cette stigmatisation continue d'imprégner nos structures, nos pratiques, nos représentations et nos attitudes. L'hétérosexisme - un "isme " relativement récent si on le compare, par exemple, au sexisme ou au racisme — fait référence à un système idéologique qui stigmatise, nie, dénigre et trouve anormal tout ce qui se trouve à l'extérieur de ce qui est perçu comme étant la norme, hétérosexuelle, prescrite socialement (Szymanski, 2008). L'hétérosexisme répandu illustre bien le chemin qu'il reste à parcourir vers la reconnaissance sociale ou le respect des minorités sexuelles et, dans une perspective de justice sociale, vers la défense des droits des personnes qui en font partie. Il est important qu'elles puissent assumer leur identité sexuelle, la légitimer aux yeux des autres, la protéger et vivre dans une société plus ouverte à leur réalité. 
Contrairement aux hommes gais qui ont acquis une plus grande visibilité et un plus grand respect en tant que groupe social (Bergeron, et collab., 2003), les femmes homosexuelles se sont trouvées à l'arrière-plan, entre autres, dans les mouvements de reconnaissance des identités sexuelles alternatives, lors de la légalisation sur les actes homosexuels et sur le mariage entre personnes de même sexe, ainsi que dans le changement des attitudes homophobes et hétérosexistes. L'histoire des femmes homosexuelles est donc moins bien connue et leur vécu se confond trop facilement à celui des hommes gais. Cela nous amène à nous demander ce que vivent de particulier les femmes homosexuelles.

La littérature consacrée aux expériences des femmes de minorité sexuelle met à jour un portrait plutôt négatif de ces dernières. Elles sont, entre autres, critiquées pour leur manque de visibilité en tant que groupe minoritaire, et cette visibilité est primordiale à leur reconnaissance sociale. Cet état de choses est largement attribuable à l'impression qu'elles ont que leurs coming out — chaque fois que leur identité sexuelle est révélée à une nouvelle personne - sont mal reçus (Bernhard, 2001). Ces femmes sont alors doublement perdantes : ou bien elles se taisent et contribuent de la sorte au manque de visibilité et de reconnaissance qu' on leur reproche (Dahan, et collab., 2008), ou bien elles vivent leurs coming out et risquent chaque fois de faire l'objet, au mieux d'hétérosexisme, au pire d'opprobre pur et simple (Van Voorhis, et collab., 2002).

Selon la littérature, et vu de l'extérieur dans ma perspective de travailleuse sociale, il est donc permis d'inférer que les femmes homosexuelles vivent des expériences négatives par rapport à leur identité et qu'elles sont sujettes à l'hétérosexisme. La littérature suggère de plus que les femmes de minorité sexuelle sont placées dans une position de "victimes ", et ce, au détriment d'un certain pouvoir de leur part.

En ce qui concerne les soins de santé, le refus de coming out auprès des professionnels expliquerait en partie un traitement qui ne tient pas compte des spécificités médicales des femmes homosexuelles (Garcia, 2003). Par ailleurs, la littérature montre 
qu'elles ne sont pas toujours adéquatement traitées à cause d'une méconnaissance de leurs spécificités ou des besoins qui leur sont particuliers (Marrazo, 2004). En effet, on ne leur reconnait aucune caractéristique à prendre en compte dans le cadre des soins qui leur sont offerts - bien entendu, une telle reconnaissance reste impossible si les femmes homosexuelles ne s'affichent pas, ou pas assez, en tant que telles (Seaver, et collab., 2008; Brotman, et collab., 2002). En somme, selon la littérature, si les femmes homosexuelles ne reçoivent pas une attention médicale adéquate, c'est parce qu'elles ne s'affichent pas assez comme minorité sexuelle et ne font pas suffisamment comprendre la spécificité des soins qu'elles sont en droit de réclamer (Kerker, et collab., 2006). Mais pourquoi s'afficher si, ce faisant, elles en sortent encore plus "victimes "? Par contre, en ne s'affichant pas, elles deviennent aussi des « victimes"

\section{Deux pôles de réflexion}

La présente réflexion se base principalement sur l'expérience et les connaissances acquises comme stagiaire et militante au sein du Centre d'aide et de lutte contre les agressions sexuelles (CALACS) de l'Outaouais. Le CALACS est un organisme communautaire dont le but est de soutenir les survivantes d'agression sexuelle.

\section{L'approche féministe}

Dans ses interventions, le CALACS préconise une approche féministe, à savoir une forme de gestion selon laquelle les intervenantes ou intervenants travaillent collectivement et sur tous les plans. Les interventions auprès des femmes reflètent les valeurs auxquelles les intervenantes ou intervenants adhèrent et qui se retrouvent dans la structure même de l'organisme. Ainsi, j'ai pu développer mes connaissances sur diverses problématiques féministes et appliquer ces valeurs lors de mes interventions. Mon expérience m'a permis de garder un positionnement honnête, transparent et égal envers les femmes victimes d'agression sexuelle, tout en gardant une certaine humilité, car je ne me présentais 
jamais à elles comme l'" experte en la matière ". Au contraire, ce sont elles qui étaient les expertes. De ce qu'elles vivaient, de ce dont elles avaient besoin.

\section{La compétence culturelle}

Les femmes de minorité sexuelle peuvent être considérées comme faisant partie d'une sous-culture au sein d'une culture générale hétérosexuelle (Peers, et collab., 1998). Cette appartenance reconnue à une sous-culture permet de dégager des caractéristiques propres aux personnes qui l'ont en partage, lesquelles caractéristiques constituent des repères fort utiles en situation d'intervention. Cette forme d'intervention modulée sur la culture de la personne rencontrée est qualifiée de «compétente culturellement ». Une approche compétente culturellement exige de l'intervenante ou intervenant d'acquérir certaines notions qui peuvent servir à comprendre le vécu de la personne, tout en évitant le piège d'associer cette dernière à une sous-culture à laquelle elle n'adhère pas dans les faits.

Les valeurs féministes auxquelles j'adhère et mon désir d'offrir aux femmes de minorité visible une intervention compétente culturellement - et d'influencer mes collègues en ce sens m'ont guidée tout au long d'un stage en milieu hospitalier. Ma première impression en fut une de déception en constatant combien la diversité — celle de minorités, qu'elles soient visibles, sexuelles ou autres - était peu reconnue, et encore moins prise en compte, dans les interventions. Or, la littérature fait état de l'importance des interventions compétentes culturellement et des dommages potentiels d'une intervention qui ignore la culture de la personne. Le fait que cette approche ne faisait pas partie de la politique de l'hôpital a bousculé mes valeurs et croyances.

Cette politique d'intervention visait en fait à considérer chaque patient comme un individu et le traiter comme nous voudrions qu'un membre de notre famille le soit. Or, je ne voyais pas comment concilier cette politique avec mon point de vue du travail social, celui d'amener sur un plan social des expériences vécues individuellement et d'ainsi extirper la personne de son 
isolement. Mais c'est en observant les autres travailleuses sociales que j'ai compris que la politique ne représentait qu'un cadre de travail. Dans ce cadre, elles parvenaient à appliquer une approche et des méthodes qui étaient empreintes des valeurs du service social qui me sont chères et que je reconnaissais dans leurs interventions.

Pour ma recherche de maitrise en service social, ce séjour en milieu hospitalier m'a poussée à explorer l'envers de la médaille, à savoir le vécu dans un contexte de soins de santé de personnes appartenant à des groupes minoritaires. Plus spécifiquement,j'ai choisi de cibler ma recherche sur l'expérience de femmes de minorité sexuelle, donc confrontées à l'hétérosexisme, cette forme d'injustice sociale encore inconnue ou mal comprise de la majorité et qui est par le fait même susceptible d'être présente dans nos institutions. Comme travailleuse sociale qui adhère à des valeurs de justice sociale et qui souhaite contribuer à l'allègement de la souffrance liée à l'oppression, il m'était très important d'accorder toute la place voulue à l'expression de ces femmes par rapport aux expériences qui leur appartiennent et qui leur sont importantes. Pour répondre à cet objectif, j'ai mené deux entrevues semidirigées lors desquelles j'ai laissé une large place à la libre parole de mes répondantes.

Bien que limités en nombre, les récits analysés ont permis de saisir en profondeur une diversité dans les choix et les préférences des femmes de minorité sexuelle. En soi, cela offre des points de repère intéressants pour aborder l'intervention. Ainsi, il sera question dans les prochains paragraphes de connaissances et d'aptitudes nécessaires à toute intervention auprès des femmes de minorité sexuelle, tout particulièrement dans un contexte de soins de santé.

\section{Les connaissances et les aptitudes}

Une intervention compétente culturellement implique l'acquisition de connaissances importantes afin de bien saisir le vécu de personnes appartenant à une culture déterminée. 


\section{Le choix des mots}

Toute intervenante ou intervenant pourrait faire face à un dilemme sémantique pour tenter de décrire, ne serait-ce qu'en partie, l'identité d'une femme de minorité sexuelle afin d'intervenir adéquatement auprès d'elle : "orientation sexuelle ", "identité sexuelle ", " lesbienne ", " gaie ", " queer "... Certains termes, "lesbienne " par exemple, sont utilisés comme reflet identitaire, alors que d'autres, telle l'expression "queer", sont susceptibles de provoquer un débat dans l'entourage. Alors, quels termes employer pour décrire cette personne, sans l'offusquer ou faire preuve d'hétérosexisme? Toute intervenante ou intervenant ne faisant pas partie d'une minorité sexuelle s'expose à un tel questionnement. De là, l'importance de développer des connaissances, ne seraientelles qu'élémentaires, sur les caractéristiques propres à cette minorité. Les notions de "nomination de soi ", de "coming out", de "visibilité " et d'" hétérosexisme " constituent un excellent point départ.

Cependant, il faut s'assurer que les connaissances préalablement acquises par l'intervenante ou intervenant tiennent compte des termes - et qu'elles ne les occultent pas - employés par les femmes pour s'identifier elles-mêmes. Car, il y a une grande diversité chez les femmes de minorité sexuelle par rapport à la nomination de soi ou à l'expression de leur identité sexuelle. Chacune choisit à sa façon les termes et modalités de divulgation de cette information, laquelle - il est bon de se le rappeler elles sont libres de divulguer ou non. Si ces femmes décident de ne pas mettre leurs interlocuteurs au courant de leur identité sexuelle, ou si elles ne le font qu'en partie, c'est qu'elles estiment à leur avantage de procéder ainsi. Et ce choix doit être respecté. Chez certaines, cela s'explique par le fait qu'elles ne veulent pas s'exposer à la discrimination basée sur l'identité sexuelle toujours présente, de façon consciente ou inconsciente, autant dans nos structures que dans nos propos. Il ne faut surtout pas juger comme problématique le choix que font certaines femmes de minorité sexuelle de taire toute information sur le sujet. Elles jugent tout simplement que cette information n'est pas pertinente ou relève du domaine privé. 


\section{Quelques qualités essentielles}

Certaines qualités et un certain savoir-être sont généralement reconnus et appréciés chez une intervenante ou intervenant auprès de femmes de minorité sexuelle. Entre autres, il y a la discrétion, tant dans le choix des termes utilisés que dans le type d'intervention menée, et l'art de développer un climat de confiance qui amène ces dernières à partager spontanément des éléments de leur identité.

Pour les femmes interviewées dans le cadre de ma recherche, il n'est pas primordial que les professionnels de la santé prennent en compte leur identité sexuelle lorsqu'elles sollicitent leurs services. Elles considèrent plutôt les qualités humaines comme étant essentielles à une intervention réussie. Un médecin, une infirmière, une physiothérapeute ou une travailleuse sociale qui se montrent sensibles et empathiques ont à leurs yeux plus de compétence dans leurs domaines que d'autres collègues. Le respect et l'écoute inhérents à toute intervention sont des qualités encore plus appréciées par la personne qui se trouve dans une position inconfortable. C'est sur cette base que se construit une relation de confiance entre elle et l'intervenante ou l'intervenant. Savoir prendre le temps est aussi une qualité fort appréciée chez l'intervenante ou intervenant.

\section{L'intervention dans le champ des soins de santé}

Il existe toujours une différence entre la personne dans son unicité et la définition que fait d'elle le groupe auquel elle appartient. Par conséquent, il importe dans le cadre d'une intervention d'éviter de renfermer la personne dans une identité qui ne lui appartient pas, de lui imposer les stéréotypes du groupe et des connaissances préalables entourant ce dernier. De plus, les qualités et expériences qui lui sont personnelles ne peuvent être occultées, ni son identité sexuelle qui est indissociable des autres aspects de son individualité. Il faut accompagner la personne avec ce qu'elle révèle au sujet d'elle-même, que cela concorde ou non avec les idées reçues. 
Enfin, en intervention, les implications sociales ne peuvent être mises de côté quand une personne choisit de dévoiler son identité sexuelle. En plus des impacts sur le groupe minoritaire, cela oblige la société en général à reconnaitre la personne dans son intégrité, et donc, de la respecter.

\section{Quelques pistes de recommandations}

En ce qui concerne les femmes de minorité sexuelle, il serait bon de réviser la formation des professionnels de la santé, afin de mesurer l'importance accordée à la prise de conscience des biais personnels dans les interventions auprès des personnes. Cela permettrait aux professionnels de la santé d'être plus outillés en vue d'interventions mieux réussies auprès de la population en général. Quant aux intervenantes et intervenants sociaux, un travail approfondi sur leurs biais personnels, pour éviter qu'ils ne se mêlent à ceux des personnes aidées, serait pertinent dans leur parcours de formation professionnelle. Chez eux, l'exercice de se remettre en question, de réévaluer leurs pratiques et de se garder toujours conscients de leurs attitudes, biais et jugements, doit être encouragé sur une base régulière, et ce, que ce soit en milieu communautaire ou institutionnel, en soins de santé ou en intervention sociale.

\section{Conclusion}

Les femmes de minorité sexuelle appartiennent à une sous-culture dont les caractéristiques sont à prendre en compte dans toute intervention dans le contexte de soins de santé. Cependant, la place qu'elles veulent bien accorder à cet aspect de leur personne peut varier d'une femme à l'autre. Pour les femmes qui ont collaboré à mon mémoire, ce n'est pas l'attention portée à leur identité sexuelle qui importe, mais plutôt les sentiments exprimés à leur égard, tels la sensibilité, l'empathie ou le respect. 
Les individus appartenant à un groupe minoritaire contribuent à en définir les caractéristiques communes. En retour, le groupe minoritaire concourt à forger la personnalité des individus qui en font partie. Cette dynamique entre la reconnaissance sociale du groupe et la reconnaissance des spécificités individuelles est certainement d'actualité pour le groupe de femmes de minorité sexuelle. Il est certes important de considérer ces dernières en fonction du groupe minoritaire auquel elles appartiennent et qui affiche ses propres spécificités. Mais d'un autre côté, il faut être en mesure d'intervenir auprès de la personne elle-même, dans son authenticité, indépendamment de ce qui peut appartenir chez elle à la minorité dont elle fait partie. De fait, la tendance de toujours vouloir catégoriser et étiqueter représente un obstacle dans la compréhension du vécu des femmes de minorité sexuelle. Bien évidemment, ce concept reste vrai pour toute personne faisant partie d'un groupe minoritaire. Cette tension entre la sphère sociale - qui encourage la reconnaissance des groupes dans une optique de justice sociale — et la sphère individuelle — qui vise à agir directement dans la vie des personnes dans le but d'alléger leur souffrance - illustre de façon éloquente certaines tensions entre différentes approches du service social. Le travailleur social ne peut évacuer les luttes sociales lorsqu'il intervient auprès d'individus dans toute leur unicité, et vice-versa. En fait, c'est la rencontre de ces deux axes qui permet le changement social et qui a un impact dans la vie des gens. Cela étant dit, c'est dans la pratique que l'on voit émerger les défis que présente cette rencontre au bout de laquelle nos structures sociales représentent de façon plus juste les personnes qui en font partie.

\section{Note}

1 GRABOWIECKA, Sabina (2010). On est comme tout le monde dans le sens qu'on n'est pas comme tout le monde : chaque personne est unique, mémoire de maitrise sous la direction de Marguerite Soulière, Université d'Ottawa. 


\section{Bibliographie}

BER GERON, Sherry, et CharleneY.SENN (2003). « Health Care Utilization in a Sample of Canadian Lesbian Women : Predictors of Risk and Resilience ", Women \& Health, Vol. 37, No 3, p. 19-35. BERNHARD, Linda A. (2001). "Lesbian health and health care ", Annual Review of Nursing Research, Vol. 19, p. 145-177.

BROTMAN, Shari, et collab. (2002). "The Impact of Coming Out on Health and Health Care Access : The Experiences of Gay, Lesbian, Bisexual and Two-Spirit People ", Journal of Health \& Social Policy, Vol. 15, № 1, p. 1-29.

DAHAN, Rachel, Rotem FELDMAN et Doron HERMONI (2008). «Is Patients'Sexual Orientation a Blind Spot of Family Physicians? ", Journal of Homosexuality, Vol. 55, No 3, p. 524-532.

GARCIA, Theresa C. (2003). "Primary care of the lesbian/gay/bisexual/transgendered woman patient ", International journal of fertility ans women's medecine, Vol. 48, № 6, p. 246-251.

KERKER, Bonnie D., Farzad MOSTASHARI et Lorna THORPE (2006). "Health Care Access and Utilization among Women Who Have Sex with Women : Sexual Behavior and Identity ", Journal of Urban Health: Bulletin of the New York Academy of Medicine, Vol. 83, No 5, p. 970-979.

KLITZMAN, Robert L., et Jason D. GREENBERG (2002). « Patterns of Communication Between Gay and Lesbian Patients and Their Health Care Providers ", Journal of Homosexuality, Vol. 42, $\mathrm{N}^{\circ} 4$, p. $65-75$.

MARRAZZO, Jeanne M. (2004). « Barriers to Infectious Disease Care among Lesbians », Emerging Infectious Diseases, Vol. 10, № 11, p. 1974-1978.

PEERS, Lynda, et Irène DEMCZUK (1998). «Lorsque le respect ne suffit pas : intervenir auprès des jeunes lesbiennes ", dans Irène Demczuk (dir.), Des droits à reconnaître. Les lesbiennes face à la discrimination, Montréal, Éditions du Remue-ménage, p. 69-127.

SAULNIER, Christine Flynn (2002). "Deciding Who to See : Lesbians Discuss Their Preferences in Health and Mental Health Care Providers ", Social Work, Vol. 47, № 4, p. 355-365.

SEAVER, Margaret R., et collab. (2008). «Healthcare Preferences Among Lesbians : A Focus Group Analysis ", Journal of Women's Health,Vol. 17, No 2, p. 215-225.

SZYMANSKI, Dawn M., Susan KASHUBECK-WEST et Jill MEYER (2008). "Internalized Heterosexism : A Historical and Theoretical Overview ", The Counselling Psychologist, Vol. 36, $\mathrm{N}^{\circ}$ 4, p. 510-524.

VANVOORHIS, Rebecca, et Marion WAGNER (2002). «Among the Missing: Content on Lesbian and Gay People in Social Work Journals », Social Work,Vol. 47, No 4, p. 345-354. 